\title{
LATTICE STUDY FOR THE TAIWAN PHOTON SOURCE
}

\author{
C.C. $\mathrm{Kuo}^{+}$, H.P. Chang, G.H. Luo, H.J. Tsai, M.H. Wang and C.T. Chen \\ National Synchrotron Radiation Research Center, Hsinchu 30076, Taiwan
}

\section{Abstract}

The feasibility study for the new $3 \mathrm{GeV}$ Taiwan synchrotron light source, dubbed Taiwan Photon Source, was initiated in July, 2004. The goal is to construct a high performance light source with extremely bright X-ray in complementary to the existing $1.5 \mathrm{GeV}$ light source in Taiwan. The ring circumference is $518.4 \mathrm{~m}$ and a 24-cell DBA lattice structure is chosen. The natural emittance with distributed dispersion is around $2 \mathrm{~nm}$-rad. A large booster ring of $499.2 \mathrm{~m}$ sharing the storage ring tunnel as well as a separated booster ring of $162 \mathrm{~m}$ are considered.

\section{INTRODUCTION}

The NSRRC has operated its $1.5 \mathrm{GeV}$ Taiwan Light Source (TLS) for more than a decade since 1993. In the past years, we have undertaken a number of enhancements such as the energy upgrade from $1.3 \mathrm{GeV}$ to $1.5 \mathrm{GeV}$, the installation of superconducting insertion devices, the replacement of the normal conducting RF cavity with a superconduting type (SRF), etc. All these works aim at the expansion of the capacity in terms of the number of beamlines, the higher photon flux as well as brilliance, and in particular the increase of photon energy in the X-ray range. As of 2004, the number of users exceeds 750 and the scientific output is increasing rapidly. However, the number of available photon ports in TLS is limited and will be fully occupied soon. Therefore, in 2004, NSRRC Board of Trustee suggested to consider the next light sources. An intermediate energy, high performance low emittance synchrotron radiation source is the most attractive facility. The ring size has been evolved from $240 \mathrm{~m}$ to more than $500 \mathrm{~m}$ during the study to allow the increase of the number of ID sections, to lower the natural emittance, and to fit into the existing NSRRC site. Finally, a ring named Taiwan Photon Source (TPS) of 518.4m, 24-cell DBA type was chosen. Figure 1 is a layout of the TLS and TPS on the NSRRC site for the same-tunnel option of the TPS booster ring.

\section{LATTICE STRUCTURE}

The goal of the machine design is to fulfill the following criteria:

Energy: $3 \sim 3.3 \mathrm{GeV}$

Brilliance: $>10^{21} \mathrm{ph} / \mathrm{sec} / \mathrm{mm}^{2} / \mathrm{mrad}^{2} / 0.1 \% \Delta \lambda / \lambda$

Emittance: $<2 \mathrm{~nm}-\mathrm{rad}$ at $3 \mathrm{GeV}$

+ cckuo@nsrrc.org.tw
The linear optical functions are tailored to meet the requirements for low natural emittance and small photon beam size, easy for injection and chromaticity correction, suitable length and number of long straights, periodicity, etc. To meet the low emittance requirement, a 24-cell DBA or TBA type is needed. We choose a double-bend type based on the constraints of the circumference and lengths of the straights. We have explored several different configurations of the lattice structures, e.g., 2-, 4-, 6-, 8-, and 24-fold symmetries as well as different lengths of IDs. We also explored an option by enlarging the drift space in the arc to allow short straights. With slightly positive dispersion in the long straights of a 24-cell DB structure, we can achieve an emittance of 1.7 nm-rad. The achromatic configuration is around 5.8 nm-rad. Such a 6 -fold symmetry configuration provides 6 long straights for injection and long IDs, and SRF modules can be in one long straight or in two standard straights. Working point is in a good region away from structure resonances. The lattice optical functions are depicted in Fig. 2, and some of the major lattice parameters are listed in Table 1. With different kind of insertion devices, the calculated brilliance with $1 \%$ emittance coupling is plotted in Fig. 3 .

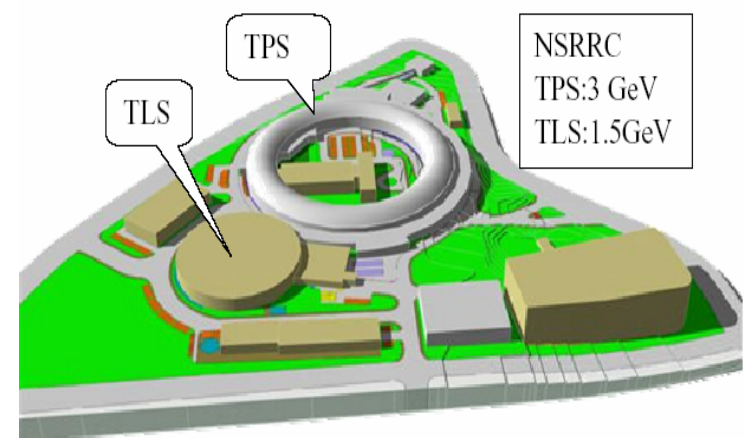

Figure 1: The TPS site plan

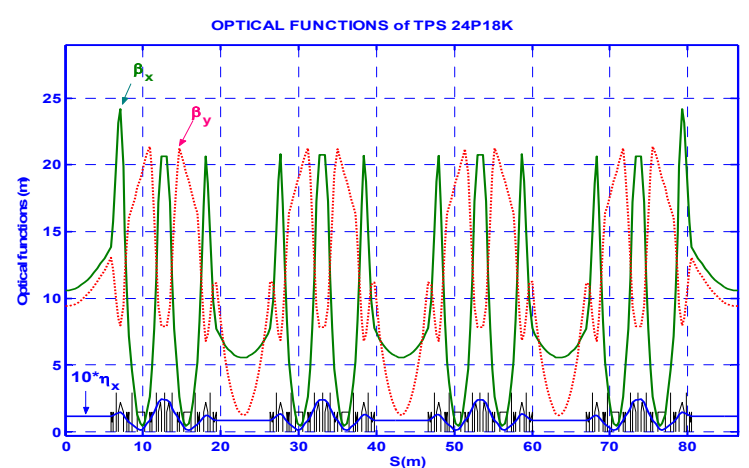

Figure 2: Optical functions of the TPS lattice. 


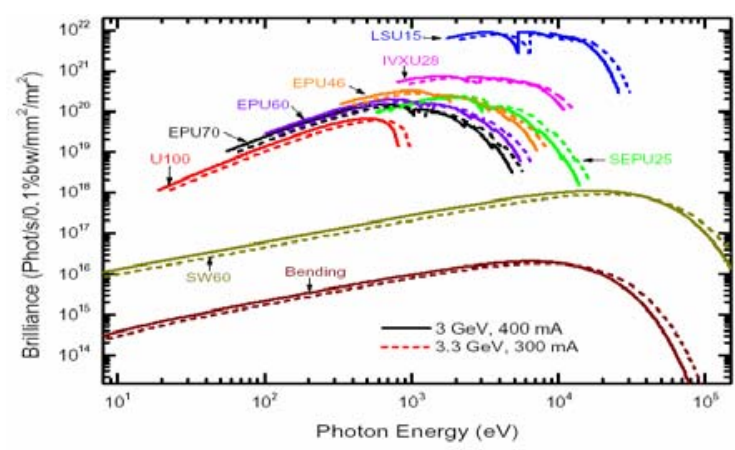

Figure 3: Brilliance of the TPS, $1 \%$ emittance ratio is assumed.

Table 1: Major parameters of the TPS

\begin{tabular}{|l|c|}
\hline Energy $(\mathrm{GeV})$ & 3.0 \\
\hline Beam current $(\mathrm{mA})$ & 400 \\
\hline Circumference $(\mathrm{m})$ & 518.4 \\
\hline Nat. emittance $(\mathrm{nm}-\mathrm{rad})$ & 1.7 \\
\hline Cell / symmetry / structure & $24 / 6 / \mathrm{DBA}$ \\
\hline$\beta_{\mathrm{x}} / \beta_{\mathrm{y}} / \eta_{\mathrm{x}}(\mathrm{m}) \mathrm{LS}$ middle & $10.59 / 9.39 / 0.11$ \\
\hline RF frequency $(\mathrm{MHz})$ & 499.654 \\
\hline RF voltage $(\mathrm{MV})$ & 5.0 \\
\hline Harmonic number & 864 \\
\hline SR loss/turn, dipole $(\mathrm{MeV})$ & 0.98733 \\
\hline Straights & $11.72 \mathrm{~m}^{*} 6+7 \mathrm{~m}^{*} 18$ \\
\hline Betatron tune $v_{\mathrm{x}} / v_{\mathrm{y}}$ & $26.22 / 12.28$ \\
\hline Synchrotron tune $v_{\mathrm{s}}$ & $6.7 \times 10^{-3}$ \\
\hline Bunch length $(\mathrm{mm})$ & 2.34 \\
\hline Dipole B/L $($ Tesla $/(\mathrm{m})$ & $1.3789 / 0.95$ \\
\hline Number of dipoles & 48 \\
\hline Quad No. / Max. field(T/m) & $240 / 17$ \\
\hline Sext No. / Max. $\mathrm{m}^{*} 1\left(\mathrm{~m}{ }^{-2}\right)$ & $168 / 6$ \\
\hline Mom. comp. $\left(\alpha_{1}, \alpha_{2}\right)$ & $2.0 \times 10^{-4}, 2.3 \times 10^{-3}$ \\
\hline Nat. energy spread $\sigma_{\mathrm{E}}$ & $9.53 \times 10^{-4}$ \\
\hline Damping partition $(\mathrm{x} / \mathrm{y} / \mathrm{s})$ & $0.997 / 1.0 / 2.003$ \\
\hline Damping time $(\mathrm{ms})(\mathrm{x} / \mathrm{y} / \mathrm{s})$ & $10.5 / 10.5 / 5.25$ \\
\hline Nat. chromaticity $\xi \mathrm{x} / \xi \mathrm{y}$ & $-78.2 /-32.5$ \\
\hline
\end{tabular}

\section{NONLINEAR OPTIMIZATION}

For a high brilliance, low emittance and low periodicity lattice structure, strong chromatic aberration induces large natural chromacticities (negative), which need to be corrected by chromatic sextupoles in the arc. The nonlinear effects driven by such strong chromatic sextupoles are detrimental, e.g., shrinkage of dynamic aperture, if not properly compensated for. It is necessary to ensure large enough dynamic aperture for the efficient injection and reasonable lifetime. The driving terms due to the chromatic sextupoles as well as quadrupoles of the off-energy particles could be reduced either by proper phase advance in the sextupole locations and/or using harmonic sextupoles. These harmonic sextupoles are carefully positioned with the suitable phase advance too. We used various popular codes for the nonlinear optimizations and for the particle tracking. These codes are OPA [1], BETA [2], PATPET [3], TRACY-2 [4], and
$\mathrm{MAD}[5]$, etc.

Basically, in the single resonance approach [6], there are 4 chromatic terms and 5 geometric terms for the first order terms and another 13 second order terms in the Hamiltonian of the single particle motion. We aim at the minimization of these terms, especially the first order terms and the terms of amplitude dependent tune shifts, and obtaining a good dynamic aperture. In the present configuration, we employ a scheme of 6-family harmonic sextupoles together with 2-family chromatic sextupoles, i.e., a scheme of 8 -family sextupoles is adopted. It is observed that dynamic aperture is very sensitive to the position of sextupoles, working point and separation of betatron functions of the linear optics. Iteration process is needed for achieving an acceptable dynamic aperture for on-energy as well as off-energy particles.

Figure 4 and 5 show the tune shift with amplitude in the horizontal and vertical planes, respectively, and Figure 6 depicts the tune shift with energy. The changes of the optical functions with energy are shown in Fig. 7.

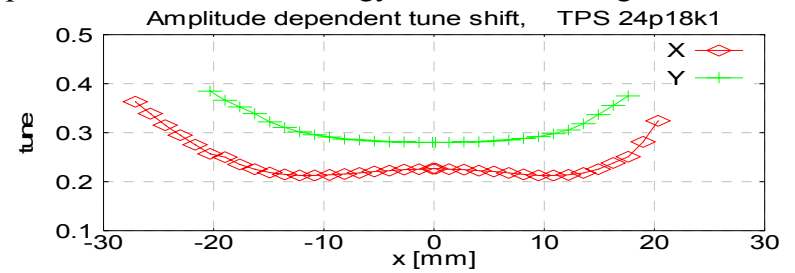

Figure 4: Tune shift vs. horizontal amplitude.

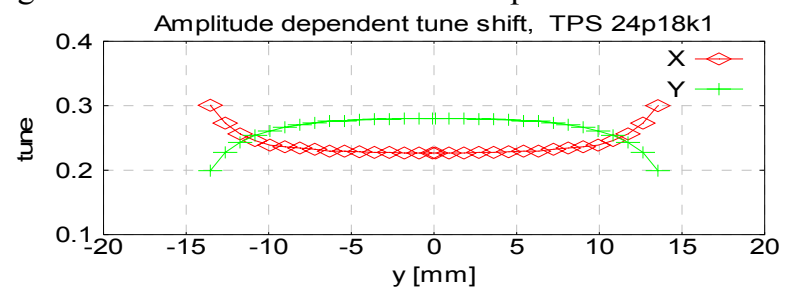

Figure 5: Tune shift vs. vertical amplitude.

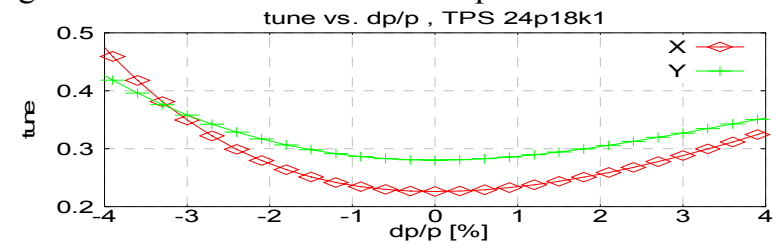

Figure 6: Tune shift vs. energy deviation.

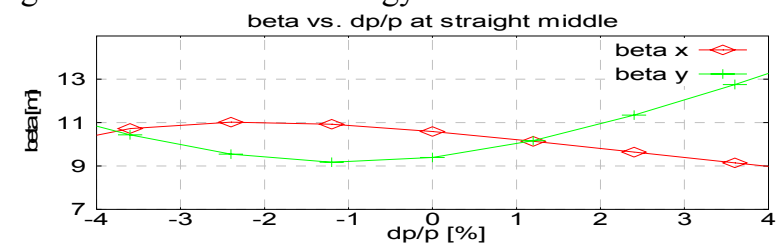

Figure 7: $\beta_{x, y}$ at long straight middle vs. energy deviation.

\section{DYNAMIC APERTURE}

With the optimized sextupole configurations, we can perform particle tracking to obtain dynamic aperture. Figure 8 gives dynamic aperture for on-energy and off-energy $( \pm 3 \%)$ particles in which synchrotron oscillation and radiation are not included. A plot for horizontal amplitude acceptance from off-energy tracking 
is shown in Fig. 9. Detailed study for a 6D tracking as well as longitudinal beam dynamics is in progress.

Other nonlinear driving sources are from the imperfection of the magnetic field in dipole, quadrupole and sextupole magnets, and from insertion devices, etc. For instance, we take a set of multipole error strengths as in [7] for this study. In Fig. 10 we can see there is some reduction of the dynamic aperture but still good enough.

There will be more than 20 insertion devices in the ring and their effects on the beam dynamics are also investigated. We put six undulators operating at NSRRC (2 EPU, 2 U5 and 2 U9) [8] in the TPS and the tracking result with these IDs is also given in Fig. 10. No significant impact on the dynamic aperture is shown.

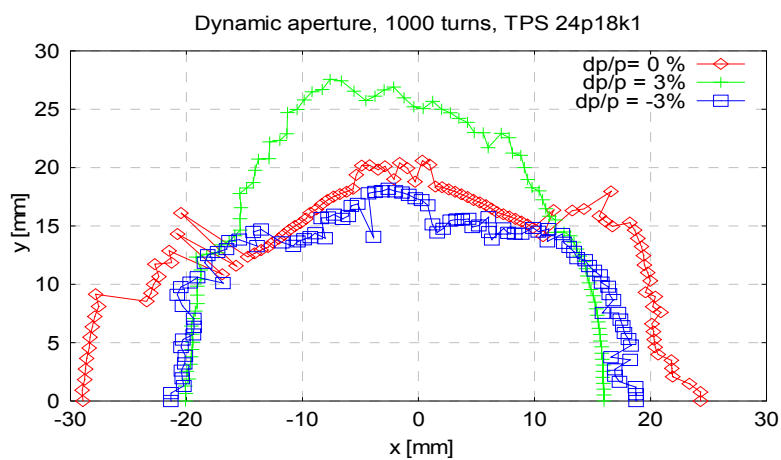

Figure 8: Dynamic aperture for on-energy and off-energy particles at long straight center.

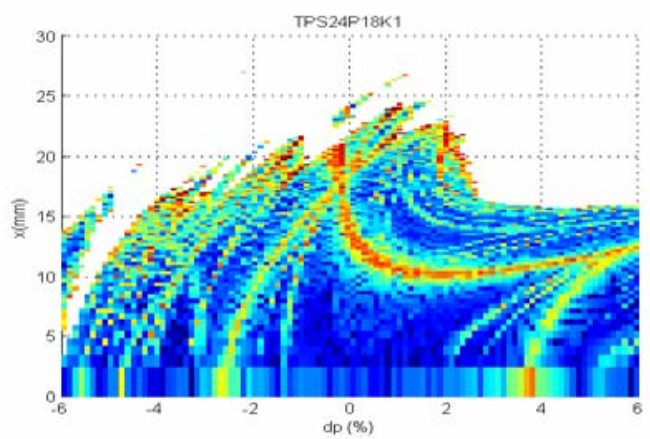

Figure 9: Horizontal dynamic aperture for off-energy particles.

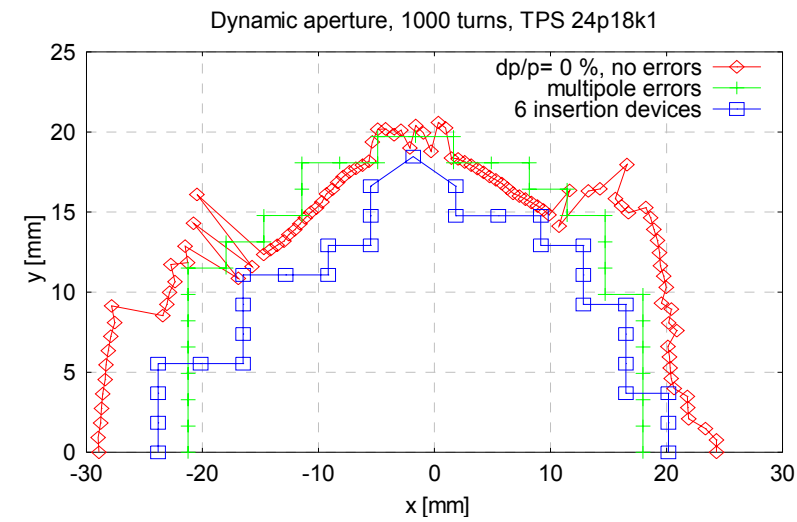

Figure 10: Dynamic aperture at long straight middle with and without multipole field errors or insertion devices.

\section{OTHER ISSUES}

We have looked into the closed orbit distortions due to misalignments of magnets, field errors, etc, and a successful correction scheme is proposed. Emittance coupling correction scheme to achieve coupling ratio $<1 \%$ is also in progress. Ground vibration issues are discussed. Accordingly, error tolerances are given. Injector booster ring sharing the same tunnel with the storage ring and a separated booster are also designed [9]. Injection scheme and aperture requirements are also studied. With successful operation experience of the SRF for the TLS[10], we will adopt same system for the TPS ring. Using SRF cavities we can alleviate longitudinal coupled-bunch instabilities. With large enough energy acceptance, we can get reasonably large Touschek lifetime and a top-up operation will be adopted. Small gap superconducting undulators need narrow aperture and the study of collective effects is under way.

\section{CONCLUSION}

We have designed a 24-cell, 6-fold symmetry, double bend lattice for the TPS, with circumference of $518.4 \mathrm{~m}$. We get a natural emittance of $1.7 \mathrm{~nm}$-rad at $3 \mathrm{GeV}$ with distributed dispersion in the long straight sections. Nonlinear optimization so far can achieve a reasonable dynamic aperture for efficient injection and reasonable lifetime. Similar results are obtained for the achromatic configuration. Further lattice optimization to meet users' need is an ongoing task.

\section{REFERENCE}

[1] A. Streun, "OPA documentation" Version 2.1, April 1997, SLS.

[2] J. Payet et al, BETA, LNS version.

[3] L. Emery, J. Safranek, H. Wiedemann, “User's Guide for PATPET version 88.2" unpublished, 1997, SLAC.

[4] J. Bengtsson, E. Forest and H. Nishimura, "Tracy User Manual", unpublished (ALS Berkely), and TRACY-2 is an SLS, SOLEIL and NSLS version.

L. Nadolski, "Application of the Frequency Map Analysis to the Study of the SOLEIL and ESRF Beam Dynamics", EPAC 2000, pp. 1060.

[5] H. Grote, F.C. Iselin, "The MAD Program", CERN/SL/90-23 (AP).

[6] J. Bengtsson, "The Sextupole Scheme for the Swiss Light Source (An Analytical Approach)", SLS Note 9/97 (1997).

[7] P. Brunelle, A. Nadji, "Performance of the Soleil Lattice in the Presence of Errors", EPAC98, pp.882.

[8] C.C. Kuo, et al, "Accelerator Physics Issues at NSRRC”, EPAC2004, pp.2377.

[9] G.H. Luo, et al, "Design Consideration of a Booster for Taiwan Photon Source", this proceedings.

[10] Ch. Wang, et al, "Operational Experience of the Superconducting Radio Frequency Cavity at NSRRC", this proceedings. 